\title{
SDSS J001641-000925: THE FIRST STABLE RED DWARF CONTACT BINARY WITH A CLOSE-IN STELLAR COMPANION
}

\author{
S.-B. Qian ${ }^{1,2,3}$, L.-Q. Jiang ${ }^{1,2,3}$, E. Fernández Lajús ${ }^{4,5,7}$, B. SoOnthornthum ${ }^{6}$, L.-Y. Zhu ${ }^{1,2,3}$, E. G. ZhaO ${ }^{1,2}$, J.-J. He $^{1,2}$, \\ W.-P. LiaO ${ }^{1,2}$, J.-J. Wang ${ }^{1,2,3}$, L. LiU ${ }^{1,2,3}$, S. Rattanasoon ${ }^{6}$, S. AukKaravittayapun ${ }^{6}$, X. Zhou ${ }^{1,2,3}$, And N. P. LiU ${ }^{1,2}$ \\ ${ }^{1}$ Yunnan Observatories, Chinese Academy of Sciences (CAS), P.O. Box 110, 650011 Kunming, China; qsb@ynao.ac.cn \\ ${ }^{2}$ Key Laboratory of the Structure and Evolution of Celestial Objects, Chinese Academy of Sciences, P.O. Box 110, 650011 Kunming, China \\ ${ }^{3}$ University of the Chinese Academy of Sciences, Yuquan Road 19\#, Sijingshang Block, 100049 Beijing, China \\ ${ }^{4}$ Facultad de Ciencias Astronómicas y Geofísicas, Universidad Nacional de La Plata, 1900 La Plata, Buenos Aires, Argentina \\ ${ }^{5}$ Instituto de Astrofisica de La Plata (CCT La plata - CONICET/UNLP), Argentina \\ ${ }^{6}$ National Astronomical Research Insititude of Thailand, 191 Siriphanich Bldg., Huay Kaew Road, Chiang Mai 50200, Thailand \\ Received 2014 October 20; accepted 2014 December 16; published 2015 January 6
}

\begin{abstract}
SDSS J001641-000925 is the first red dwarf contact binary star with an orbital period of 0.19856 days that is one of the shortest known periods among M-dwarf binary systems. The orbital period was detected to be decreasing rapidly at a rate of $\dot{P} \sim 8 \mathrm{~s} \mathrm{yr}^{-1}$. This indicated that SDSS J001641-000925 was undergoing coalescence via a dynamical mass transfer or loss and thus this red dwarf contact binary is dynamically unstable. To understand the properties of the period change, we monitored the binary system photometrically from 2011 September 2 to 2014 October 1 by using several telescopes in the world and 25 eclipse times were determined. It is discovered that the rapid decrease of the orbital period is not true. This is contrary to the prediction that the system is merging driven by rapid mass transfer or loss. Our preliminary analysis suggests that the observed minus calculated $(O-C)$ diagram shows a cyclic oscillation with an amplitude of 0.00255 days and a period of $5.7 \mathrm{yr}$. The cyclic variation can be explained by the light travel time effect via the presence of a cool stellar companion with a mass of $M_{3} \sin i^{\prime} \sim 0.14 M_{\odot}$. The orbital separation between the third body and the central binary is about $2.8 \mathrm{AU}$. These results reveal that the rarity of red dwarf contact binaries could not be explained by rapidly dynamical destruction and the presence of the third body helps to form the red dwarf contact binary.
\end{abstract}

Key words: binaries: close - binaries: eclipsing - stars: evolution - stars: individual (SDSS J001641-000925)

\section{INTRODUCTION}

W UMa-type binary stars are composed of two cool mainsequence stars where both components are in contact with each other and share a common convective envelope (CCE). This kind of binary stars under the short-period limit (0.22 days) are very rare (e.g., Rucinski 1992; Becker et al. 2011). Up to now, only a few contact binaries below the limit were discovered, e.g., GSC 0137-00475 with a period of 0.2178 (Rucinski \& Pribulla 2008), and only one red dwarf contact binary, SDSS J001641-000925, with a period of 0.1985615 days, was detected by Davenport et al. (2013), although M dwarfs are the most numerous stars in the Galaxy. The rarity of contact binaries below this limit could be explained by the following reasons: (1) they are difficult to produce because the timescale of the angular momentum loss (AML) is much longer (e.g., Stepień 2006, 2011); (2) they are still very difficult to detect because of their faintness; and (3) these systems are so unstable that they are rapidly destroyed (e.g., Jiang et al. 2012). Few contact binaries were detected from the WFCAM (Wide-Field Camera) Transit Survey (WTS) with the $3.8 \mathrm{~m}$ United Kingdom Infrared Telescope (UKIRT; e.g., Nefs et al. 2012). This indicates that their faintness is not an issue. At the same time, the discovery of several potential contact systems below the limit implies that the AML timescales have been overestimated for M dwarfs (e.g., Stepień 2011), while timescales by unstable mass transfer are too short to explain the observations (e.g., Jiang et al. 2012).

\footnotetext{
7 Visiting Astronomer, Complejo Astronómico El Leoncito operated under agreement between the Consejo Nacional de Investigaciones Científicas y Técnicas de la República Argentina and the National Universities of La Plata, Córdoba and San Juan.
}

Recently, some close binaries with periods under the limit $(P<0.22$ days) were discovered by several surveys (e.g., SDSS, WTS, and SuperWASP; e.g., Becker et al. 2011; Nefs et al. 2012; Lohr et al. 2013). As pointed out by Qian et al. (2014a), observations and investigations of those close binaries can provide valuable information on the origin and evolution of contact binaries as well as on the formation and migration history of low-mass stars. By monitoring some eclipsing binaries photometrically, we could obtain information on the changes of the orbital period that could give some constrains on the formation and the evolutionary states of contact binaries. The analysis of the light travel time effect by using eclipse times could provide information on the triplicity and then help to understand third-body interactions in the birth environment. As one of recently detected close binaries under the period limit, SDSS J001641-000925 was discovered as a faint EWtype eclipsing binary by the Sloan Digital Sky Survey (SDSS; Becker et al. 2011) of spectral type $M_{0}-M_{1}$. The light variation of the EW-type binary is continuous and the depths of the two minima are nearly the same. These properties reveal tidally distorted components and both components have similar temperature. It is an extremely short-period binary system with a period below 0.2 days ( 0.1985615 days). The first photometric and spectroscopic investigations by Davenport et al. (2013) suggest that it is a contact binary system with $M_{1}=0.58$ and $M_{2}=0.34 M_{\odot}$. The authors pointed out that the orbital period is decreasing rapidly. This is in agreement with the prediction proposed by Jiang et al. (2012) that the system is dynamically unstable and undergoing active coalescence. These properties suggest that SDSS J001641-000925 is a key target to test the theories and to study the properties of red dwarf contact binaries. 

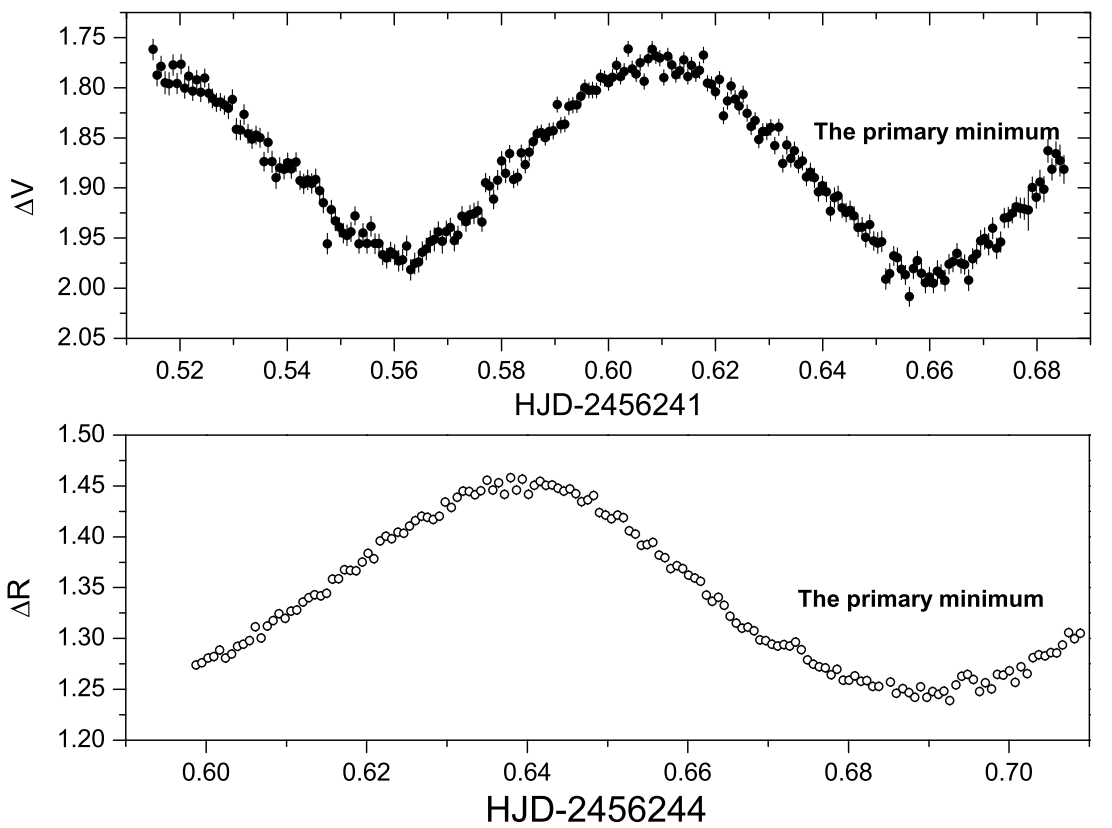

Figure 1. Light curves of SDSS J001641-000925 obtained by using the $2.15 \mathrm{~m}$ "Jorge Sahade" telescope in Argentina. The light curve in the $V$ band (solid dots) observed on 2012 November 9 is shown in the upper panel, while the $R$-band light curve (open circles) obtained on 2012 November 12 is displayed in the lower panel.

\section{PHOTOMETRIC MONITORING AND CONSTRUCTING THE $O-C$ DIAGRAM}

After the discovery of the EW-type eclipsing binary SDSS J001641-000925 by Becker et al. (2011), a rapid decrease in the $O-C$ diagram was reported by Davenport et al. (2013). To confirm the rapid decrease in the orbital period, we have monitored the eclipsing binary photometrically for determining times of minimum light. This binary was observed by using the $2.4 \mathrm{~m}$ telescope at GaoMeiGu observational station of Yunnan Observatories (YNOs) in 2011 September and November and in 2013 September. The Yunnan Faint Object Spectrograph and Camera (YFOSC), which has a $2 \mathrm{~K} \times 4 \mathrm{~K}$ camera, attached to the $2.4 \mathrm{~m}$ telescope was used. During the observation, the $R$ and $I$ filters, which are close to the Johnson's standard photometric system, were used. This binary was later monitored in 2012 and 2014 with Roper Scientific, the Versarray 1300B camera, with a thinned EEV CCD36-40 de $1340 \times 1300$ pixel CCD chip, attached to the $2.15 \mathrm{~m}$ "Jorge Sahade" telescope at Complejo Astronomico El Leoncito (CASLEO), San Juan, Argentina. The clock of the control computer operating the VersArray 1300B CCD camera is calibrated against UTC time by the GPS receiver's clock. All images were reduced by using PHOT (measure magnitudes for a list of stars) of the aperture photometry package of IRAF. The light curves in the $V$ and $R$ bands obtained in 2012 November with the $2.15 \mathrm{~m}$ "Jorge Sahade" telescope are shown in Figure 1. It is shown that the light curves are typical EW-type. The light varies continuously and has a very small difference between the depths of the two minima. It is seen in the figure that the light curve at the eclipses is symmetric and therefore times of minimum light were determined with a parabolic fitting method, i.e., a two-degree polynomial was used to fit each observed light minimum. The time standard used for the data is Heliocentric Julian Dates (HJDs) and the times were not corrected for the barycentric correction.

To get more data, this EW-type binary was then monitored continuously by using several telescopes, i.e., the $1.0 \mathrm{~m}$ telescope in the main part of YNOs, the $85 \mathrm{~cm}$ and the $2.16 \mathrm{~m}$ telescopes in the Xinglong station of national astronomical observatories (NAOs), and the $2.4 \mathrm{~m}$ telescope of national astronomical research institute in Thailand (NARIT). In total, 25 times of minimum light were obtained and are shown in Table 1. Those shown in the third column are the filters used during the observations where " $\mathrm{N}$ " indicates that no filters were used. The telescopes used for the observations are displayed in the fourth column of Table 1 where " $2.4 \mathrm{~m}$ " represents the $2.4 \mathrm{~m}$ telescope in GaoMeiGu observational station of YNOs, while " $2.15 \mathrm{~m}$ " refers to the $2.15 \mathrm{~m}$ "Jorge Sahade" telescope in Argentina. " $85 \mathrm{~cm}$ " and " $2.16 \mathrm{~m}$ " represent the $85 \mathrm{~cm}$ and $2.16 \mathrm{~m}$ telescopes in the Xinglong station of NAO. "Thai $2.4 \mathrm{~m}$ " refers to the $2.4 \mathrm{~m}$ telescope in Thailand, while " $1.0 \mathrm{~m}$ " to the $1.0 \mathrm{~m}$ telescope of YNOs.

Photometric observations in the $g, r, i$, and $z$ bands were obtained by Davenport et al. (2013) from the New Mexico State University (NMSU) $1 \mathrm{~m}$ telescope and were kindly provided to us by Dr. James R. A. Davenport. By using those data, some times of minimum light were determined and are also listed in Table 1 . The $O-C$ values of all available times of minimum light were computed with the following linear ephemeris,

$$
\text { Min. } \mathrm{I}(\mathrm{HJD})=2456241.66006+0^{\mathrm{d}} .198561 \times E,
$$

where 2456241.66006 is the initial epoch which is one of our eclipse times shown in Table 1, while 0.198561 days is the orbital period that was determined by Davenport et al. (2013) with those griz photometric data. The corresponding $O-C$ diagram is shown in Figure 2 along with the epoch number $E$ where solid dots refer to the NMSU data from Davenport et al. (2013), while open circles represent our new observations. The SDSS data were not used since they are so sparsely sampled. Davenport et al. (2013) reported that the orbital period is decreasing rapidly at A rate of $\dot{P} \sim 8 \mathrm{~s} \mathrm{yr}^{-1}$. For comparison, this rate of decrease in the orbital period is also plotted in the figure as dashed line. As we can see, the dashed line did not follow the observations, indicating that that the rapid decrease in the orbital period is not true. 
Table 1

New Available CCD Times of Minimum Light

\begin{tabular}{|c|c|c|c|}
\hline $\begin{array}{l}\text { HJD } \\
\text { (days) }\end{array}$ & $\begin{array}{l}\text { Errors } \\
\text { (days) }\end{array}$ & Filters & Telescopes \\
\hline 2455513.13813 & 0.00099 & $g$ & NMSU $1 \mathrm{~m}$ \\
\hline 2455513.23698 & 0.00199 & $g$ & NMSU $1 \mathrm{~m}$ \\
\hline 2455513.13785 & 0.00145 & $i$ & NMSU $1 \mathrm{~m}$ \\
\hline 2455513.23731 & 0.00177 & $i$ & NMSU $1 \mathrm{~m}$ \\
\hline 2455513.13756 & 0.00088 & $r$ & NMSU $1 \mathrm{~m}$ \\
\hline 2455513.23609 & 0.00122 & $r$ & NMSU $1 \mathrm{~m}$ \\
\hline 2455513.13762 & 0.00175 & $z$ & NMSU $1 \mathrm{~m}$ \\
\hline 2455513.23765 & 0.00118 & $z$ & NMSU $1 \mathrm{~m}$ \\
\hline 2455519.19170 & 0.00160 & $i$ & NMSU $1 \mathrm{~m}$ \\
\hline 2455522.17067 & 0.00121 & $r$ & NMSU $1 \mathrm{~m}$ \\
\hline 2455523.16460 & 0.00096 & $z$ & NMSU $1 \mathrm{~m}$ \\
\hline 2455526.14305 & 0.00076 & $g$ & NMSU $1 \mathrm{~m}$ \\
\hline 2455526.24177 & 0.00205 & $g$ & NMSU $1 \mathrm{~m}$ \\
\hline 2455527.13604 & 0.00090 & $g$ & NMSU $1 \mathrm{~m}$ \\
\hline 2455528.22865 & 0.00055 & $r$ & NMSU $1 \mathrm{~m}$ \\
\hline 2455531.10770 & 0.00073 & $r$ & NMSU $1 \mathrm{~m}$ \\
\hline 2455531.20729 & 0.00062 & $r$ & NMSU $1 \mathrm{~m}$ \\
\hline 2455532.19997 & 0.00049 & $r$ & NMSU $1 \mathrm{~m}$ \\
\hline 2455807.20963 & 0.00040 & $I$ & $2.4 \mathrm{~m}$ \\
\hline 2455807.20961 & 0.00070 & $R$ & $2.4 \mathrm{~m}$ \\
\hline 2455876.11066 & 0.00047 & $I$ & $2.4 \mathrm{~m}$ \\
\hline 2455876.10989 & 0.00055 & $R$ & $2.4 \mathrm{~m}$ \\
\hline 2455877.20104 & 0.00046 & $I$ & $2.4 \mathrm{~m}$ \\
\hline 2455877.19908 & 0.00058 & $R$ & $2.4 \mathrm{~m}$ \\
\hline 2455878.19351 & 0.00036 & $I$ & $2.4 \mathrm{~m}$ \\
\hline 2455878.19392 & 0.00044 & $R$ & $2.4 \mathrm{~m}$ \\
\hline 2456241.66006 & 0.00044 & $V$ & $2.15 \mathrm{~m}$ \\
\hline 2456241.56010 & 0.00044 & $V$ & $2.15 \mathrm{~m}$ \\
\hline 2456242.65429 & 0.00067 & $I$ & $2.15 \mathrm{~m}$ \\
\hline 2456242.55466 & 0.00035 & $I$ & $2.15 \mathrm{~m}$ \\
\hline 2456243.64551 & 0.00039 & $R$ & $2.15 \mathrm{~m}$ \\
\hline 2456244.63920 & 0.00034 & $R$ & $2.15 \mathrm{~m}$ \\
\hline 2456893.73705 & 0.00026 & $R$ & $2.15 \mathrm{~m}$ \\
\hline 2456893.83641 & 0.00031 & $R$ & $2.15 \mathrm{~m}$ \\
\hline 2456895.72246 & 0.00024 & $R$ & $2.15 \mathrm{~m}$ \\
\hline 2456895.82087 & 0.00024 & $R$ & $2.15 \mathrm{~m}$ \\
\hline 2456575.14445 & 0.00009 & $N$ & $2.4 \mathrm{~m}$ \\
\hline 2456513.29223 & 0.00025 & $N$ & $85 \mathrm{~cm}$ \\
\hline 2456550.22481 & 0.00099 & $N$ & $85 \mathrm{~cm}$ \\
\hline 2456895.32669 & 0.00058 & $N$ & $85 \mathrm{~cm}$ \\
\hline 2456667.97069 & 0.00044 & $N$ & $2.16 \mathrm{~m}$ \\
\hline 2456658.04367 & 0.00013 & $N$ & Thai $2.4 \mathrm{~m}$ \\
\hline 2456932.25759 & 0.00103 & $N$ & $1.0 \mathrm{~m}$ \\
\hline
\end{tabular}

\section{THE CYCLIC CHANGE OF THE $O-C$ DIAGRAM AND THE PRESENCE OF A THIRD BODY}

As shown in Figure 3, the linear ephemeris of SDSS J001641000925 needs to be revised, and it appears that there is a cyclic variation as well. To describe the general trend of the $O-C$ curve satisfactorily, a combination of a revised linear ephemeris and an additional cyclic variation is required. A least-squares solution yields the following equation

$$
\begin{aligned}
O-C= & 0.00049( \pm 0.00009) \\
& +0.00000135( \pm 0.00000021) \times E \\
& +0.00255( \pm 0.00065) \sin [0.0344( \pm 0.0010) \times E \\
& \left.+176^{\circ} .4( \pm 0.9)\right] .
\end{aligned}
$$

The first value in the right-hand side of the equation (0.00049) is the revision of the initial epoch, while 0.00000135 is the revision of the orbital period. By using the method proposed by

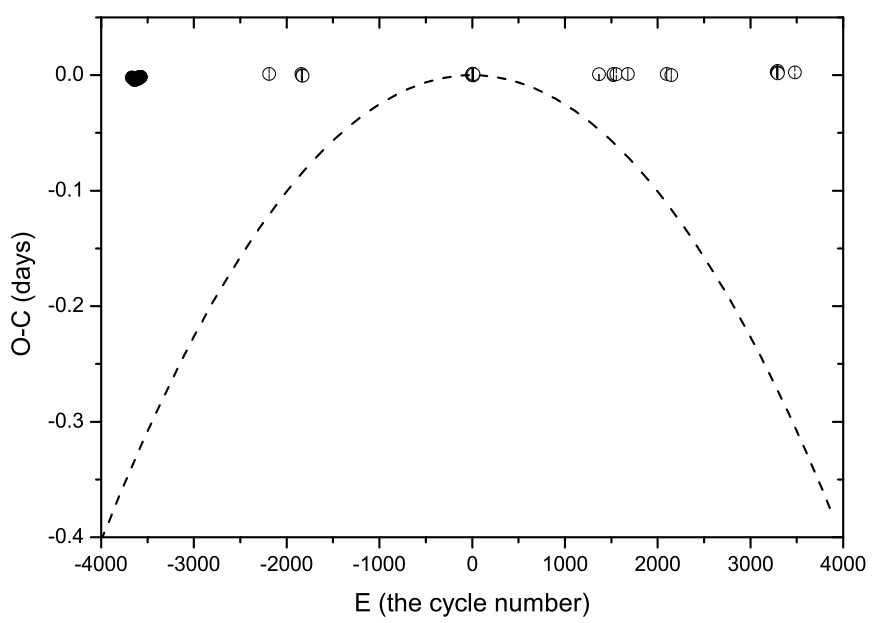

Figure 2. $O-C$ diagram of SDSS J001641-000925 with respect to the linear ephemeris in Equation (1). To construct the diagram, 43 newly determined eclipse times were used. The dashed line represents the rapid decrease in the orbital period predicted by Davenport et al. (2013). Solid dots refer to the data from Davenport et al. (2013) and open circles to our new data. It is shown that the observations do not follow the general trend of the rapid decrease.
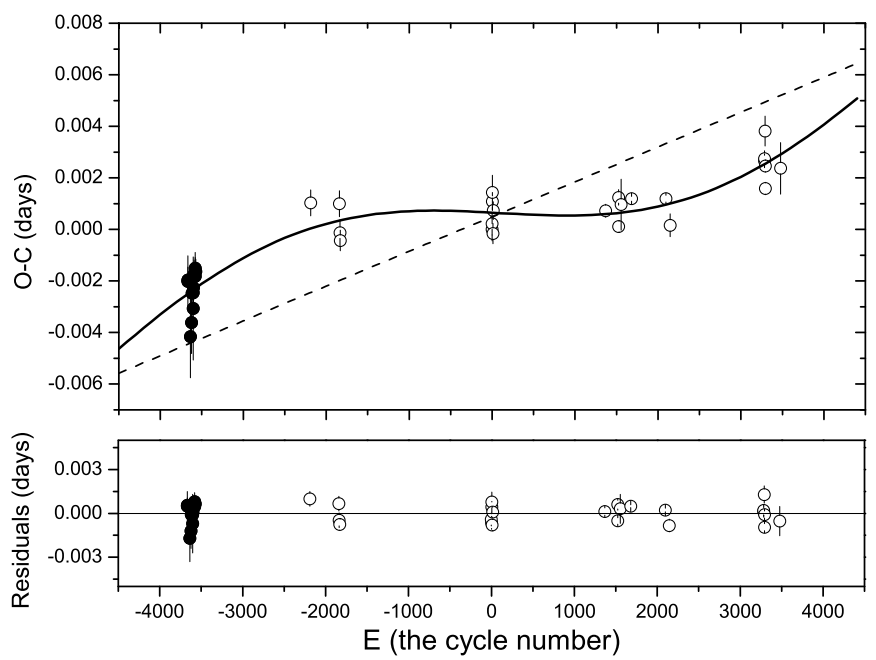

Figure 3. Cyclic change of the $O-C$ diagram. The dashed line refers to a revised linear ephemeris, while the solid line refers to the combination of the cyclic change and the revised ephemeris. Symbols are the same as those in Figure 2. The residuals after the revised period and the cyclic variation are removed from the $\mathrm{O}-\mathrm{C}$ curve are displayed in the lower panel.

Pringle (1975), the significance of the cyclic fit was tested. The calculated $\lambda$ value suggests that the cyclic change is significant at more than the $99.99 \%$ level. Then the following revised linear ephemeris,

$$
\begin{aligned}
\text { Min. } I= & 2456241.66055( \pm 0.00009) \\
& +0.19856253( \pm 0.00000021) \times E,
\end{aligned}
$$

was obtained. The derived orbital period is 0.19856253 days, which is slightly longer than that obtained by Davenport et al. (2013). The dashed line in the upper panel of Figure 3 refers to the revised ephemeris, while the solid line in the same panel to the combination of the new linear ephemeris and the cyclic change.

The cyclic oscillation has an amplitude of 0.00255 days. By using $P_{3}=\left(360^{\circ} / w\right) \times P$, the period of the cyclic variation is determined to be $P_{3}=2079.3( \pm 69.4)$ days $=5.69( \pm 0.19) \mathrm{yr}$, where $w=0.0344( \pm 0.0010)$ is the frequency. The residuals from Equation (2) are displayed in lower panel of Figure 3, 
Table 2

Parameters of the Tertiary Component in SDSS J001641-000925

\begin{tabular}{llc}
\hline \hline Parameters & \multicolumn{1}{c}{ Values } & Units \\
\hline$P_{3}$ & $5.69( \pm 0.19)$ & Years \\
$A_{3}$ & $0.00255( \pm 0.00065)$ & Days \\
$e^{\prime}$ & 0.0 & Assumed \\
$a_{12}^{\prime} \sin i^{\prime}$ & $0.44( \pm 0.10)$ & A.U. \\
$f(m)$ & $2.7( \pm 0.9) \times 10^{-3}$ & $M_{\odot}$ \\
$M_{3} \sin i^{\prime}$ & $0.144( \pm 0.037)$ & $M_{\odot}$ \\
$a_{3}\left(i^{\prime}=90^{\circ}\right)$ & $2.8( \pm 0.9)$ & A.U. \\
\hline
\end{tabular}

where no variations can be traced, indicating that Equation (2) describes the general $O-C$ trend well.

As shown in Figure 3, both the primary and the secondary times of minimum light follow the same general trend of the $O-C$ change: the orbit of the binary should be circular. This suggests that the oscillation in the $O-C$ curve is not caused by apsidal motion via an eccentric orbit of the binary star. This is in agreement with the extremely short orbital period. As discussed by several authors (e.g., Liao \& Qian 2010; Qian et al. 2013a, $2013 \mathrm{~b}$ ), this kind of cyclic variation can plausibly be explained by the light travel time effect (LTTE). Therefore, we analyze the cyclic change for LTTE via the presence of a tertiary component. As displayed in Figure 3, the sine-like change of the $\mathrm{O}-\mathrm{C}$ curve reveals that the eccentricity of the orbit of the tertiary component is close to zero. With the same method used by Qian (e.g., Qian et al. 2013a, 2013b), the parameters of the third body are derived. During the computation, we assumed that the third body is moving in a circular orbit, i.e., the eccentricity $e^{\prime}=0.0$. The projected radius of the orbit of the central eclipsing pair $\left(a_{12}^{\prime} \sin i^{\prime}\right)$ rotating around the mass central of the triple system was calculated with the equation

$$
a_{12}^{\prime} \sin i^{\prime}=A_{3} \times c,
$$

where $A_{3}$ is the amplitude of the $O-C$ oscillation, $c$ the speed of light, and $i^{\prime}$ is the orbital inclination of the third body. Then, a computation with the following equation

$$
f(m)=\frac{4 \pi^{2}}{G P_{3}^{2}} \times\left(a_{12}^{\prime} \sin i^{\prime}\right)^{3},
$$

leads to a mass function of $f(m)=2.7( \pm 0.9) \times 10^{-3} M_{\odot} . G$ and $P_{3}$ in Equation (5) are the gravitational constant and the period of the $\mathrm{O}-\mathrm{C}$ oscillation. Finally, by using the parameters derived by Davenport et al. (2013), the masses $\left(M_{3}\right)$ and the orbital separations $\left(a_{3}\right)$ of the third body for different values of $i^{\prime}$ could be calculated by the use of the following equation

$$
f(m)=\frac{\left(M_{3} \sin i^{\prime}\right)^{3}}{\left(M_{1}+M_{2}+M_{3}\right)^{2}} .
$$

All of the parameters of the tertiary companion are shown in Table 2.

\section{DISCUSSIONS AND CONCLUSIONS}

The absolute lower limit on the orbital period for contact binary star systems is an unsolved question in stellar astrophysics. Jiang et al. (2012) showed that dynamically unstable mass transfer for low-mass contact binaries may result in rapid coalescence and the observed period limit would thus be explained as a result of the very short lifetimes for such binary systems. The orbital period of SDSS J001641-000925 was detected to decay rapidly at a rate of $\dot{P} \sim 8 \mathrm{~s} \mathrm{yr}^{-1}$, which seems to support this idea.
Based on the newly determined times of minimum light listed in Table 1, we discovered that the rapid decrease in the orbital period is not true and the $\mathrm{O}-\mathrm{C}$ diagram may show a acyclic change with a period of $5.7 \mathrm{yr}$ and an amplitude of 0.00255 days. This result suggests that SDSS J001641-000925 is the first stable red dwarf contact binary revealing that the short-period limit of contact binaries could not be explained by rapidly dynamical destruction. Contact binaries under the short-period limit are rare because they are difficult to produce and/or are still very difficult to detect (e.g., Qian et al. 2014a).

The cyclic change in the $O-C$ diagram may suggest that it is triple system with a cool stellar companion with a mass of $M_{3} \sin i^{\prime} \sim 0.14 M_{\odot}$ at an orbital separation of about $2.8 \mathrm{AU}$. Similar close-in companions to contact binaries have been reported, such as PY Vir with a companion at an separation of 2.8 AU (Zhu et al. 2013a), V401 Cyg with a companion at an orbital distance of about 3.0 AU (Zhu et al. 2013b), and CSTAR 038663 with a close-in companion at an orbital separation of $0.93 \mathrm{AU}$ (Qian et al. 2014b). The extremely low masses of both components in SDSS J001641-000925 suggests that the formation of the contact binary is driving by AML via magnetic braking. However, since the timescale of the AML for the two red dwarf components is very long (e.g., Stepień 2006, 2011), the close-in stellar companion should play an important role for the origin and evolution by removing angular momentum from the central pair during the early stellar dynamical interaction or late evolution that causes the binary to have a very low angular momentum and a very short initial orbital period. In that case, can the initially detached shortperiod, red dwarf progenitor evolves into contact configuration via angular momentum loss from the magnetic stellar wind (e.g., Qian et al. 2007a, 2007b, 2013a). It is possible that all very short period M-dwarf binaries (such as in Nefs et al. 2012) are actually triple systems. Continuously photometric monitoring of those eclipsing binaries are needed to check their triplicity.

The work is partly supported by the Chinese Natural Science Foundation (Nos. 11133007 and 11325315). New observations were obtained with the $2.4 \mathrm{~m}$ and $1.0 \mathrm{~m}$ telescopes in YNOs and the $85 \mathrm{~cm}$ and the $2.16 \mathrm{~m}$ telescopes at the Xinglong station of NAOs in China, the $2.15 \mathrm{~m}$ "Jorge Sahade" telescope in Argentina, and the $2.4 \mathrm{~m}$ telescope of NARIT. The authors thank Dr. James R. A. Davenport for sending photometric observations in the $g, r, i$, and $z$ bands.

\section{REFERENCES}

Becker, A. C., Bochanski, J. J., Hawley, S. L., et al. 2011, ApJ, 731, 17 Davenport, J. R. A., Becker, A. C., West, A. A., et al. 2013, ApJ, 764, 62 Jiang, D.-K., Han, Z.-W., Ge, H.-W., et al. 2012, MNRAS, 421, 2769 Liao, W.-P., \& Qian, S.-B. 2010, MNRAS, 405, 1930

Lohr, M. E., Norton, A. J., Kolb, U. C., et al. 2013, A\&A, 549, 86 Nefs, S. V., Birkby, J. L., Snellen, I. A. G., et al. 2012, MNRAS, 425, 950 Pringle, J. E. 1975, MNRAS, 170, 633

Qian, S.-B., Jiang, L.-Q., Zhu, L.-Y., et al. 2014a, CoSka, 43, 290

Qian, S.-B., Liu, N-P., Li, K., et al. 2013a, ApJS, 209, 13

Qian, S.-B., Wang, J.-J., Zhu, L.-Y., et al. 2014b, ApJS, 212, 4

Qian, S.-B., Xiang, F.-Y., Zhu, L.-Y., et al. 2007a, AJ, 133, 357

Qian, S.-B., Yuan, J.-Z., Xiang, F.-Y., et al. 2007b, AJ, 134, 1769

Qian, S.-B., Zhang, J., Wang, J.-J., et al. 2013b, ApJS, 207, 22

Rucinski, S. M. 1992, AJ, 103, 960

Rucinski, S. M., \& Pribulla, T. 2008, MNRAS, 388, 1831

Stepień, K. 2006, AcA, 56, 199

Stepień, K. 2011, AcA, 61, 139

Zhu, L. Y., Qian, S. B., Liu, N. P., Liu, L., \& Jiang, L. Q. 2013a, AJ, 145, 39

Zhu, L.-Y., Qian, S.-B., Zhou, X., et al. 2013b, AJ, 146, 28 